\title{
CORONAL DISTURBANCES OBSERVED IN THE OPTICAL EMMISSION LINES
}

\author{
M. WALDMEIER
}

Swiss Federal Observatory, Zürich, Switzerland

\begin{abstract}
Some examples are presented of fast variations of the structure and the intensity of coronal condensations. For the observations the lines $\lambda 5303$ and $\lambda 5694$ have been used. Most of the observed phenomena are flare-connected, but all of them are related to some kind of $\mathrm{H} \alpha$-activity. Sudden brightening of the emission lines may occur after the disappearance of a filament. The events have a duration of up to several hours and consist of hot clouds of high density that are slowly rising from the upper chromosphere into the lower corona.
\end{abstract}

Coronal disturbances can optically best be observed in the lines 5303 and $5694 \AA$. The first is reasonably intense and the second visible only in the disturbance itself.

The monochromatic corona is mostly quiet. In the course of hours generally one observes but gradual variations of the line intensities. Even variations from day to day are small and mostly due to the solar rotation. Most of the features can be followed on several consecutive days. In this respect the corona has some similarity with the stationary filaments. These are formations in the innermost corona and undergo very slow evolutions like the corona.

Under coronal disturbance we understand variations of the intensity or the structure of the corona that take place in a time not longer than a few hours. Practically all these disturbances are in close connection with centers of activity and most of them in connection with a special $\mathrm{H} \alpha$-activity in that center. If no $\mathrm{H} \alpha$-activity is seen by observing the coronal disturbance, it may be that the $\mathrm{H} \alpha$-activity is not at the limb but on the disk or behind the limb. From observations over 35 years, I got the impression that all coronal disturbances are in connection with some kind of $\mathrm{H} \alpha$ activity. The relation between the importance of a coronal disturbance and the importance of the responsible chromospheric phenomenon is poor. Most of the flares are of importance 1, having little height and not reaching the level that is observed by coronagraphs. These flares in general are not accompanied by coronal disturbances. There are a few exceptions when small flares have produced noticeable coronal disturbances. Larger flares which penetrate higher into the corona always produce disturbances, especially when they are followed by surges or loops. Surges not connected with flares and ascending prominences may or may not affect the corona. Other activities not flare connected, like active prominences streaming down into a center of attraction, may also disturb the corona.

The nature of these disturbances may consist of:

(a) fluctuations of the intensity;

(b) expansions of already existing structures, especially arches;

(c) disruptions of existing structures;

(d) ejections; or

(e) formation of new structures. 
Most of the coronal disturbances have a sporadic character. In general, when the disturbance is over, the corona looks very much the same as before. As Lyot (1944) has noticed already $30 \mathrm{yr}$ ago, the variations of the structure of the corona are not arising from mass motions, but from brightening of some regions and fading of others. This is certainly true for the slow events. The phenomena we are calling disturbances are fast events, and in these as a rule mass motion is involved. Doppler shifts of the emission lines indicate mass motion in the corona. If the spectrogram shows chromospheric lines too, these lines exhibit Doppler shifts of the same sign as the coronal lines, but in general not of the same amount as the coronal features; even if they appear at the same place, they are spaced from each other along the line of sight.

After these general remarks we will present some examples.

\section{Disturbance by Ascending Prominences}

A rising prominence was observed at heliographic latitude north $45^{\circ}$ on the east limb on April 9, 1956. It was violent ascent with Doppler shifts corresponding to $300 \mathrm{~km} \mathrm{~s}^{-1}$. The distribution of the intensity of the line $\lambda 5303$ was observed one hour before the ascent began and again half an hour after the disappearance of the prominence. Not the slightest change of the structure or the intensity of the green line was noticed. So it seems that chromospheric material may move through the corona without affecting it. This example is not conclusive as the rising filament was not at the limb. Therefore, the region where the filament crossed the line of sight was rather far above the Sun's limb and did contribute but little to the total line intensity. Even if this region was affected by the filament, the influence upon the line intensity could hardly be detected.

Another example is more conclusive. On March 18, 1961, a formerly stable filament at latitude north $27^{\circ}$ on the east limb was rising. The intensity distribution of the line $\lambda 5303$ immediately before the ascent did not show anything unusual. It was almost the same as on the day before, and it was still unchanged after the disappearance of the filament. Two hours after the filament had vanished, the region, occupied before by the stable prominence, became suddenly very bright, much brighter than the maxima over the main zones of activity (Waldmeier, 1961). The red line $\lambda 6374$ also became very bright at the same time. Two arches, not very regular in shape, were formed, the outer reaching a height of $100000 \mathrm{~km}$ and the lower one $50000 \mathrm{~km}$. The region of the inner arch showed the line $\lambda 5694$. This is quite unusual, and is the only case where this line has been observed in a spotless region. Two hours later, the arches had disappeared, but the now structureless region was still unusually bright. It was only about six hours after the appearance of the condensation that it had faded away completely. This phenomenon has some similarity with the behaviour of the chromosphere in connection with rising filaments. It was on September 2, 1937 that the author for the first time observed a flare-like brightening about half an hour after the dissolution of a filament (Waldmeier, 1938). It is by now well known 
that the sudden disappearance of a filament is, not always but often, followed by flare-like $\mathrm{H} \alpha$ emission. Bruzek (1957) has given several examples. The brightening of the corona after a 'disparition brusque' is unique. I have observed this phenomenon only once.

\section{Disturbance by a Flare}

On September 21, 1955, the whole development of a relatively small but very bright limb flare and of the coronal disturbance connected with it was observed (Figure 1). The line $\lambda 5303$ began to brighten at the beginning of the flare. The brightening of this line was still going on when, after $15 \mathrm{~min}$, the flare faded away. The maximum brightness occurred about one hour after the outbreak of the flare. The intensity

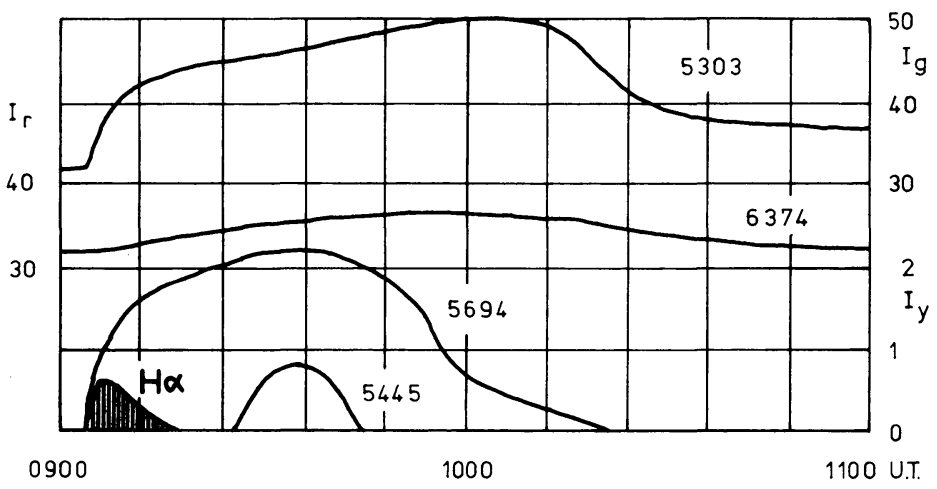

Fig. 1. The variations of the intensities of the green, $I_{g}$, the red, $I_{r}$, and the two yellow lines, $I_{\gamma}$, in connection with a small flare on September 21, 1955 at latitude south $25^{\circ}$ on the west limb.

decreased slowly returning to its preflare value only after three hours. The line $\lambda 6374$ was little affected. It showed a gradual rise and fall, with a maximum 40 min after the beginning of the flare. A strong reaction was observed in the line $\lambda 5694$. At the beginning of the flare the intensity of this line, that was not visible before, rose sharply, reached its maximum half an hour later and became invisible 70 min later. At its maximum, when the flare had already disappeared, the second line of $\mathrm{Ca} \mathrm{xv}, \lambda 5445$, became visible for $15 \mathrm{~min}$. Out of the flare, a cloud of hot gases was emerging at a low speed of a few $\mathrm{km}$ per second. Half an hour after the flare it reached its highest temperature at a height of about $25000 \mathrm{~km}$. In the center of the hot cloud, gases condensed into bright $\mathrm{H} \alpha$ knots. The hot cloud persisted for about three hours. This hot cloud, originating from a flare, may be responsible for the postburst increase of the microwave radiation. The microwave brightness temperature of the hot cloud is about one hundred times that of the undisturbed solar disk, but its area is one thousand times smaller than that of the Sun. Therefore, the postburst increase should be of the order of $10 \%$ of the preflare intensity.

The formation of hot clouds, actually the hottest regions of the corona, is a charac- 
teristic disturbance following a flare. During its formation, the cloud is rising and becoming hotter. The hottest points are not found closest to the limb but at an altitude of roughly $50000 \mathrm{~km}$ (Figure 2).

A similar event was observed on August 14, 1970. At the latitude north $9^{\circ}$ on

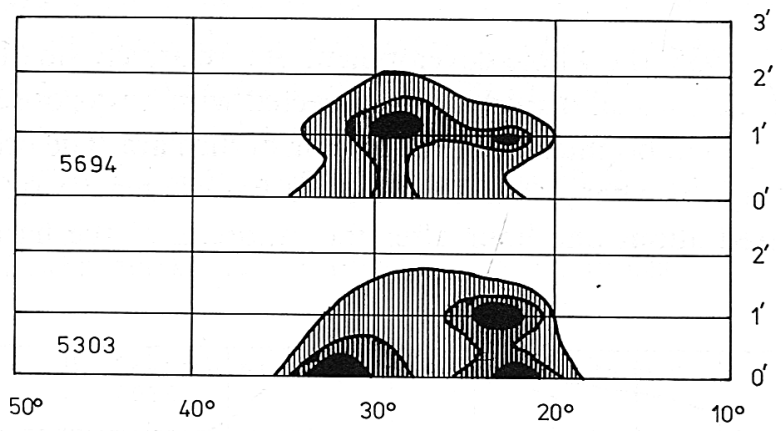

Fig. 2. A hot cloud condensation, observed on April 1, 1957 at 1120 UT at the west limb. Heights are given in arc minutes above the edge of the occulting disk. The knots visible only in the line $\lambda 5303$ are low lying and of relatively low temperature. The knot at latitude north $29^{\circ}$, visible only in the line $\lambda 5694$ at a height of $50000 \mathrm{~km}$, is the hottest feature in the condensation.

the east limb, the appearance of the line $\lambda 5694$ indicated that a strong center of activity was approaching the limb. At that time, a big spot of type $\mathrm{H}$ was one day behind the limb. The bright but quiet corona became suddenly active in that place. The region of the $\lambda 5694$ emission was strongly increasing in intensity and rising. Half an hour later, the hot cloud reached its greatest brightness at a level of $40000 \mathrm{~km}$ above the limb. At that time, according to observations in $H \alpha$ and $D_{3}$, loops were fully developed. In the line $\lambda 5303$ the structure became very complex. The appearance of the corona in this line agreed only in the major features with that in the line $\lambda 5694$ or with the $\mathrm{H} \alpha$ loops. The whole phenomenon is so characteristic for a postflare event that there must have been a flare behind the limb.

\section{What is First, the Hot Cloud or the Flare?}

The two examples I have mentioned cannot answer this question. In the first one, the intensities of both the chromospheric and the coronal lines were rising simultaneously, and in the second one, the flare occurred behind the limb. Active centers likely to produce flares are always surrounded by a permanent coronal condensation. If it is large and dense enough, it emits the line $\lambda 5694$; therefore it is hot. Such a hot cloud could become the origin of a flare, or a flare may produce the hot cloud. The material now available indicates clearly that the flare precedes the formation of hot coronal clouds.

On March 18, 1956, at 1015 UT, a small flare appeared at the latitude north $28^{\circ}$ 
on the east limb (Figure 3). The spectrum was carefully watched from the beginning. The chromospheric lines were extremely bright, but the line $\lambda 5303$ was unchanged compared to the preflare intensity while $\lambda 5694$ was invisible. At 1035 UT the flare came to end. It was only at that moment, and not before, that the coronal lines became affected. The line $\lambda 5694$ was first seen at $1040 \mathrm{UT}$, reached its highest in-

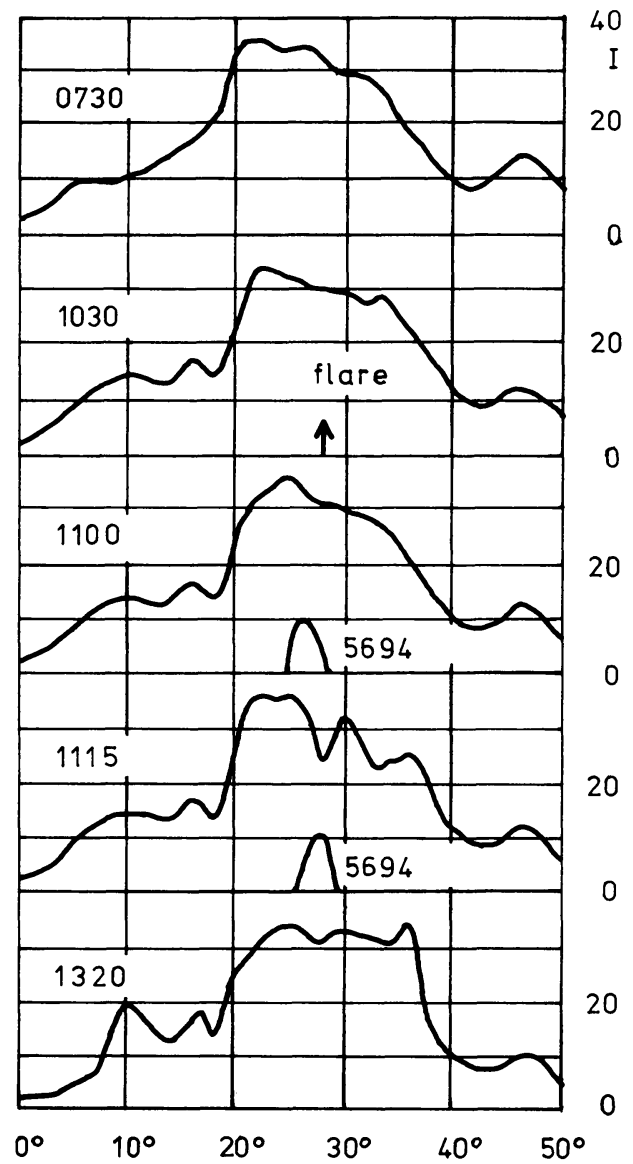

Fig. 3. The variations of the intensities of the lines $\lambda 5303$ and $\lambda 5694$ following a flare at latitude north $28^{\circ}$ on the east limb on March $18,1956$.

tensity around $1100 \mathrm{UT}$, and disappeared at $1130 \mathrm{UT}$. At the time of the visibility of this line, the green line was strongly weakened. Two hours after the flare, the line intensities were the same as before the flare.

This example, one out of many others, shows clearly that the hot coronal cloud comes after the flare, and has a long duration. We do not conclude that the coronal cloud is produced by the optical flare. Probably, both the flare and the cloud have a common primary origin that is not visible, and not yet known. 


\section{Conclusions}

Disturbances of the corona that take place in a time not longer than a few hours, are rare phenomena. The variations in most of these fast events consist of changes both in structure and intensity but mass motion is also involved in many of them. They always are connected with flares or some other $\mathrm{H} \alpha$ activity. The most remarkable optical features are a very large intensity of the line $\lambda 5694$ and a strong continuoum, indicating high temperature and density. Typically the highest, densest and hottest condensations occur not close to the Sun's limb but at a height of about $40000 \mathrm{~km}$.

\section{References}

Bruzek, A.: 1957, Z. Astrophys. 42, 76.

Lyot, B.: 1944, Ann. Astrophys. 7, 31.

Waldmeier, M.: 1938, Z. Astrophys. 15, 310.

Waldmeier, M.: 1961, Z. Astrophys. 53, 206.

\section{DISCUSSION}

Altschuler: What mechanism forms the yellow line region?

Waldmeier: I have only presented observational results; but we might assume that they are related to the X-ray regions. 